TRANSACTIONS OF THE

AMERICAN MATHEMATICAL SOCIETY

Volume 360, Number 12, December 2008, Pages 6389-6402

S 0002-9947(08)04622-9

Article electronically published on July 22, 2008

\title{
FIXED POINT PROPERTY AND THE FOURIER ALGEBRA OF A LOCALLY COMPACT GROUP
}

\author{
ANTHONY TO-MING LAU AND MICHAEL LEINERT
}

\begin{abstract}
We establish some characterizations of the weak fixed point property (weak fpp) for noncommutative (and commutative) $\mathcal{L}^{1}$ spaces and use this for the Fourier algebra $A(G)$ of a locally compact group $G$. In particular we show that if $G$ is an IN-group, then $A(G)$ has the weak fpp if and only if $G$ is compact. We also show that if $G$ is any locally compact group, then $A(G)$ has the fixed point property (fpp) if and only if $G$ is finite. Furthermore if a nonzero closed ideal of $A(G)$ has the fpp, then $G$ must be discrete.
\end{abstract}

\section{INTRODUCTION}

Let $E$ be a Banach space and $K$ be a nonempty bounded closed convex subset of $E$. We say that $K$ has the fixed point property (or simply fpp) if every nonexpansive mapping $T: K \rightarrow K$ (i.e., $\|T x-T y\| \leq\|x-y\|$ for all $x, y \in K$ ) has a fixed point. We say that $E$ has the (resp. weak) fixed point property if every bounded closed (resp. weakly compact) convex subset $K \subseteq E$ has the fixed point property.

It is well known that $\ell^{1}$ has the weak fixed point property (see for example [28]) but not the fpp (see [19]). A well known result of Browder [7] asserts that if $E$ is uniformly convex, then $E$ has the weak fpp. Kirk [18] extended this result by showing that if $K$ is a weakly compact convex subset of $E$ with normal structure, then $K$ has the fpp. As shown by Alspach [1] (see also [3. Theorem 4.2] and [4) the Banach space $L^{1}[0,1]$ does not have the weak fpp (and hence not the fpp). In fact, he exhibited a weakly compact convex subset $K$ of $L^{1}[0,1]$ and an isometry $T: K \rightarrow K$ $(\|T x-T y\|=\|x-y\|$ for all $x, y \in K)$ without a fixed point. In particular $A(\mathbb{Z}) \cong L^{1}(\mathbb{T})$ does not have the weak fpp nor the fpp where $\mathbb{T}=\{\lambda \in \mathbb{C} ;|\lambda|=1\}$ is the circle group with multiplication and $\mathbb{Z}$ is the additive group of the integers. On the other hand, as seen above $A(\mathbb{T}) \cong \ell^{1}(\mathbb{Z})$ has the weak fpp but not the fpp.

Let $G$ be a locally compact group and $A(G)$ be its Fourier algebra. $G$ is called an [IN]-group if there is a compact neighbourhood $U$ of the identity such that $x^{-1} U x=U$ for all $x \in G$. [IN]-groups properly include all groups in which the left and right uniformities agree. It was shown by Lau and Mah [21, Theorem 5] that if $G$ is a compact group, then $A(G)$ has weak ${ }^{*}$-normal structure and hence the weak fpp. More recently, Lau, Mah and Ülger [23] show that the converse is also true if

Received by the editors November 10, 2006.

2000 Mathematics Subject Classification. Primary 43A15, 47A09; Secondary 43A20, 47H10, $46 \mathrm{~B} 22$.

Key words and phrases. Weak fixed point property, nonexpansive mapping, Fourier algebra, noncommutative $\mathcal{L}^{1}$ space, semifinite von Neumann algebra.

The research of the first author was supported by NSERC Grant A-7679.

(C)2008 American Mathematical Society 
$G$ is either the direct product of a compact group and an abelian locally compact group, or $G$ is a connected [IN] group. In this paper, we shall prove (Corollary 4.2) among other things, that if $G$ is an [IN]-group, then $A(G)$ has the weak fpp if and only if $G$ is compact. In particular, if $G$ is a discrete group, then $A(G)$ has the weak fpp if and only if $G$ is finite. This is proved by using noncommutative integration with respect to a trace on a von Neumann algebra. We also include some results from commutative integration.

For $A(\mathbb{Z}) \cong \mathcal{L}^{1}(0,1)$ it is known (see [19, p. 52]) that a closed subspace has the fpp if and only if it is reflexive. We consider nonzero closed ideals in $A(G)$ for a locally compact group $G$ and show that if such an ideal has the fpp, then $G$ must be discrete. We also show that if $A(G)$ has the fpp, then $G$ must be finite. A fortiori, this also holds for the Fourier-Stieltjes algebra $B(G)$.

We shall use, among other things, results from [36]. There is, however, a problem in Remark 3.3 of [36] due to the fact that for a positive linear functional $\phi$ on a von Neumann algebra $\mathcal{M}$ and a projection $P \in \mathcal{M}$ the functional $\phi \cdot P$ is not necessarily positive. As a consequence, there are gaps in the proofs of Theorems 3.4 and 3.5 in 36. We therefore give modified versions for the remark and these theorems:

[36, Remark 3.3*]: Suppose $\tau$ is a normal trace on $\mathcal{M}$ and $Q \in \mathcal{M}$ is a projection with $\tau(Q)<\infty$. The functional $\phi: A \mapsto \tau(Q A Q)$ (when extended from $\mathcal{M}^{+}$to $\mathcal{M})$ is a positive element of $\mathcal{M}_{*}$. If a projection $P \in \mathcal{M}$ commutes with $Q$, we have $\phi P \geq 0$ and $\|\phi P\|=\phi P(I)=\tau(Q P Q)=\phi(P)$.

[36, Theorem 3.4*] is [36, Theorem 3.4] with the additional assumption that $\mathcal{M}$ be semifinite.

Proof. Suppose $\tau$ is a faithful semifinite normal trace on $\mathcal{M}$ and $R \in \mathcal{M}$ is a projection which dominates no minimal projection. As in the first few lines of the proof of Lemma 3.1, there is a projection $Q \leq R$ with $0<\tau(Q)<\infty$, and we may assume $\tau(Q)=1$, so $\phi: A \mapsto \tau(Q A Q)$ is a normal state on $\mathcal{M}$ (when extended to be linear continuous) with $\phi(Q)=1$. Now continue as in the proof of Theorem 3.4 of [36] with $P$ replaced by $Q$. In the course of this, use [36, Remark $3.3^{*}$ ] to see that $\|g(t)-g(s)\|=2|t-s|$.

[36. Theorem $3.5^{*}$ ] is [36, Theorem 3.5] with the statement " $\mathcal{M}$ is semifinite" added under point (c). The proof is as before, using [36, Theorem $\left.3.4^{*}\right]$ instead of [36, Theorem 3.4].

In the text below, when we refer to $3.3,3.4$, or 3.5 of [36], we mean $3.3^{*}, 3.4^{*}$, or $3.5^{*}$, respectively.

Let us also suggest (even though this is not relevant for our paper) that in 36, Theorem 4.1, (d)] and [36, Theorem 4.2, (d)] one should add " $V N(G)$ is semifinite" and " $\mathcal{M}(G)$ is semifinite", respectively.

The second named author would like to thank Michael Cowling for fruitful discussions.

\section{Some PRELIMINARIES}

All topologies considered in this paper are assumed to be Hausdorff.

Let $K$ be a bounded closed convex subset of a Banach space $E$. A point $x$ in $K$ is called a diametral point if

$$
\sup \{\|x-y\|: y \in K\}=\operatorname{diam}(K),
$$


where diam $(K)$ denotes the diameter of $K$. The set $K$ is said to have normal structure if every nontrivial (i.e., contains at least two points) convex subset $H$ of $K$ contains a nondiametral point of $H$. A Banach space has weak-normal structure if every nontrivial weakly compact convex subset has normal structure.

A Banach space $E$ is said to have property UKK (uniformly Kadec-Klee property) if for any $\varepsilon>0$ there is a $0<\delta<1$ such that whenever $\left(x_{n}\right)$ is a sequence in the unit ball of $E$ converging weakly and satisfying $\inf \left\{\left\|x_{n}-x_{m}\right\|: n \neq m\right\}>\varepsilon$, then $\left\|\lim x_{n}\right\| \leq \delta$ (see [17]). As known [13], if $E$ has property UKK, then $E$ has the weak normal structure and hence also the fixed point property.

A Banach space $E$ is said to have the Radon Nikodym property (= RNP) if each closed convex subset $D$ of $E$ is dentable i.e., for any $\varepsilon>0$, there exists an $x$ in $D$ such that $x \notin \overline{\mathrm{co}}\left(D \backslash B_{\varepsilon}(x)\right)$, where $B_{\varepsilon}(x)=\{y \in X:\|x-y\|<; \varepsilon\}$ and $\overline{\mathrm{co}} K$ is the closed convex hull of a set $K \subseteq E$. (See [5].)

A Banach space $E$ is said to have the Krein-Milman Property (= KMP) if every closed bounded convex subset of $E$ is the closed convex hull of its set of extreme points.

A measure space $(X, \mathcal{F}, \mu)$ is called localizable if the canonical monomorphism from $\mathcal{L}^{\infty}(\mu)$ into the dual space of $\mathcal{L}^{1}(\mu)$ is surjective (hence an isomorphism). An element $B \in \mathcal{F}$ is called a $\mu$-atom if $\mu(B)>0$ and $\mu(C)=0$ or $\mu(C)=\mu(B)$ for every $C \subset B, C \in \mathcal{F}$.

A weight on a measure space $(X, \mathcal{F}, \mu)$ is a measurable function $\omega: X \rightarrow[0, \infty]$. By $\omega \mu$ we then mean the measure defined by $\omega \mu(A)=\int_{A} \omega d \mu$ for $A \in \mathcal{F}$.

Let $H$ be a Hilbert space, and let $\mathcal{A}$ be a von Neumann algebra on $H, \mathcal{A}^{+}$be the set of positive operators in $\mathcal{A}$, and $\mathcal{A}^{\prime}$ be its commutant. Let $\tau: \mathcal{A}^{+} \rightarrow[0, \infty]$ be a trace, i.e., a function on $\mathcal{A}^{+}$satisfying

(i) $\tau(\lambda A)=\lambda \tau(A)$ for all $\lambda \geq 0, A \in \mathcal{A}^{+}$(with $0 \cdot \infty=0$ ),

(ii) $\tau(A+B)=\tau(A)+\tau(B), A, B \in \mathcal{A}^{+}$,

(iii) $\tau\left(A^{*} A\right)=\tau\left(A A^{*}\right)$ for all $A \in \mathcal{A}$.

The trace $\tau$ is called faithful if $\tau(A)=0, A \in \mathcal{A}^{+}$, implies $A=0 ; \tau$ is semifinite if $\tau(A)=\sup \left\{\tau(B) \mid B \in \mathcal{A}^{+} ; B \leq A, \tau(B)<\infty\right\}$; and $\tau$ is normal if for any increasing net $\left(A_{\alpha}\right)$ in $\mathcal{A}^{+}$with $A_{\alpha} \uparrow A$, in the weak*-topology $=\sigma=\sigma\left(\mathcal{A}, \mathcal{A}_{*}\right)$ topology, where $\mathcal{A}_{*}$ is the unique predual of $\mathcal{A}$, one has $\tau\left(A_{\alpha}\right) \uparrow \tau(A)$.

Let $\tau$ be a semifinite normal trace on $\mathcal{A}$; let $s(\tau)$ be the support projection of $\tau$, i.e., $s(\tau)$ is a central projection in $\mathcal{A}$ such that $\tau=0$ on $(I-s(\tau)) \mathcal{A}^{+}$and $\tau$ is faithful on $s(\tau) \mathcal{A}$. Let

$$
\begin{gathered}
J_{\tau}=\left\{A \in s(\tau) \mathcal{A} ; \tau\left(A^{*} A\right)<\infty\right\}, \\
\mathcal{A}_{\tau}=\left\{\sum_{i=1}^{n} A_{i} B_{i} ; x_{i}, y_{i} \in J_{\tau}\right\} .
\end{gathered}
$$

Then $\mathcal{A}_{\tau}$ is an ideal in $s(\tau) \mathcal{A}$, and $A \in \mathcal{A}_{\tau}$ if and only if its absolute value $|A|=$ $\left(A^{*} A\right)^{1 / 2}$ belongs to $\mathcal{A}_{\tau}$. For $A \in \mathcal{A}_{\tau}$, define

$$
\|A\|_{1}=\tau(|A|) ;
$$

then $\left(\mathcal{A}_{\tau},\|\cdot\|_{1}\right)$ is a normed linear space which is isometrically isomorphic to a subspace of the predual space $\mathcal{A}_{*}$ by the map $\phi: \mathcal{A}_{\tau} \rightarrow s(\tau) \cdot \mathcal{A}_{*}, A \mapsto \phi_{A}$, where for $A \in \mathcal{A}_{\tau}$,

$$
\phi_{A}(B)=\tau(B A), \quad B \in A \quad \text { and } \quad(s(\tau) \cdot \psi)(B)=\psi(s(\tau) B), \psi \in \mathcal{A}_{*} .
$$


Let $L^{1}(\mathcal{A}, \tau)$ denote the completion of $\left(\mathcal{A}_{\tau},\|\cdot\|_{1}\right)$. Since $\left\{\phi_{A} ; A \in \mathcal{A}_{\tau}\right\}$ is dense in $s(\tau) \cdot \mathcal{A}_{*}$, the linear isometry $\phi$ extends to an isometric isomorphism of $L^{1}(\mathcal{A}, \tau)$ onto $s(\tau) \cdot \mathcal{A}_{*}=(s(\tau) \cdot \mathcal{A})_{*}$.

\section{Noncommutative $L^{1}$ AND WEAK FPP}

The following lemma is a simple consequence of [36], Lemma 3.2:

Lemma 3.1. Let $\mathcal{A}$ be a von Neumann algebra, and $\tau$ be a faithful semifinite normal trace $\tau$. If there is a nonzero projection $P$ in $\mathcal{A}$ which dominates no minimal projection, then $L^{1}(\mathcal{A}, \tau)$ contains an isometric copy of $L^{1}[0,1]$.

Proof. Since $\tau$ is semifinite, there exists a nonzero element $A \in \mathcal{A}^{+}$such that $A \leq P$ and $\tau(A)<\infty$. By the spectral decomposition theorem, there exists a scalar $\lambda>0$ and a nonzero projection $Q$ such that $\lambda Q \leq P$. We then have $\tau(Q) \leq\left(\frac{1}{\lambda}\right) \tau(A)<\infty$ and $Q \leq P$. Consequently we may assume (by replacing $\tau$ by $\frac{1}{\tau(Q)} \tau$ ) that $\tau(Q)=1$, and $Q$ also dominates no minimal projection. So $\tau$ restricted to the von Neumann algebra $Q \mathcal{A} Q$ is a normal state. By Lemma 3.2 of [36], there exists a projection valued measure $E \rightarrow P_{E}$ from $B$ into the projections in $\mathcal{A}$ such that $P_{[0,1]}=Q$, $\left\langle\tau, P_{E}\right\rangle=\lambda(E)$ for any $E \in B$, where $B$ denotes the sigma-algebra of Borel subsets of $[0,1]$, and $\lambda$ is the Lebesque measure on $[0,1]$. Clearly, for each $E \in B, P_{E} \in$ $L^{1}(\mathcal{A}, \tau)$. Let $\rho[0,1] \subseteq L^{1}[0,1]$ denote the space of simple measurable functions on $[0,1]$. Suppose $f=\sum_{i=1}^{n} \lambda_{i} 1_{E_{i}} \in \rho[0,1]$; define $\Phi(f)=\sum_{i=1}^{n} \lambda_{i} P_{E_{i}}$. Then $\Phi$ is a welldefined linear map defined on $\rho[0,1]$ such that $\|\Phi(f)\|_{1}=\|f\|_{1}$. Hence $\Phi$ extends to a linear isometry from $L^{1}[0,1]$ into $L^{1}(\mathcal{A}, \tau)$.

Remark 3.2. a) The lemma in particular implies that, under the given assumptions, $L^{1}(\mathcal{A}, \tau)$ does not have the weak fpp.

b) In the commutative case, the lemma means that for any localizable measure space $(X, \mathcal{F}, \mu)$ with $\mu(A)=\sup \{\mu(B) \mid B \subset A, B \in \mathcal{F}, \mu(B)<\infty\}$ for all $A \in \mathcal{F}$, if some $A \in \mathcal{F}$ with $\mu(A)>0$ does not contain any $\mu$-atom $B \in \mathcal{F}$, then $\mathcal{L}^{1}(X, \mathcal{F}, \mu)$ contains an isometric copy of $L^{1}[0,1]$. In this special case we can actually do a little better, dropping the semifiniteness condition and the localizability assumption:

Proposition 3.3. Let $(X, \mathcal{F}, \mu)$ be a measure space. If there is some $A \in \mathcal{F}$ with $\mu(A)>0$ which does not contain any $\mu$-atom, then $\mathcal{L}^{1}(X, \mathcal{F}, \mu)$ contains an isometric copy of $L^{1}[0,1]$. In particular, $\mathcal{L}^{1}(X, \mathcal{F}, \mu)$ does not have the weak fixed point property.

Proof. By assumption, $A$ itself is not a $\mu$-atom, so there is some $A^{\prime} \subset A, A^{\prime} \in \mathcal{F}$, with $0<\mu\left(A^{\prime}\right)<\mu(A)$. Therefore, replacing $A$ by $A^{\prime}$, we may assume that $\mu(A)<\infty$. If we denote $\{F \cap A \mid F \in \mathcal{F}\}$ by $\mathcal{F} \cap A$, then $\mathcal{L}^{1}\left(A, \mathcal{F} \cap A, \frac{1}{\mu(A)} \mu\right)$ contains an isometric copy of $\mathcal{L}^{1}[0,1]$. This can be seen with the help of Caratheodory's characterization of nonatomic separable normalized measure algebras without atoms (see [15, p. 173]) and an argument involving the construction of a "coarser" $\sigma$ algebra $\mathcal{F}^{1} \subset \mathcal{F} \cap A$, so that $\left(A, \mathcal{F}^{1}, \frac{1}{\mu(A)} \mu\right)$ is separable (see [15, p. 168]). Since $f \mapsto \mu(A) \cdot f$ is an isometric isomorphism from $\mathcal{L}^{1}\left(A, \mathcal{F}^{1}, \mu\right)$ onto $\mathcal{L}^{1}\left(A, \mathcal{F}^{1}, \frac{1}{\mu(A)} \mu\right)$ and $\mathcal{L}^{1}\left(A, \mathcal{F}^{1}, \mu\right)$ is a closed subspace of $\mathcal{L}^{1}(A, \mathcal{F}, \mu)$, this latter space contains an isometric copy of $\mathcal{L}^{1}[0,1]$. 
Let $\tau$ be a trace on a von Neumann algebra $\mathcal{A}$. We say that $\mathcal{A}$ is $\tau$-atomic if every projection $P$ with $\tau(P)>0$ dominates a minimal projection.

From Lemma 3.1 and known facts we obtain

Proposition 3.4. Let $\mathcal{A}$ be a von Neumann algebra, and $\tau$ be a normal semifinite trace on $\mathcal{A}$. The following are equivalent:

(1) $L^{1}(\mathcal{A}, \tau)$ has the Radon Nikodym property.

(2) $L^{1}(\mathcal{A}, \tau)$ has the Krein Milman property.

(3) $L^{1}(\mathcal{A}, \tau)$ has UKK.

(4) $L^{1}(\mathcal{A}, \tau)$ has weak normal structure.

(5) $L^{1}(\mathcal{A}, \tau)$ has the weak fixed point property.

(6) $L^{1}(\mathcal{A}, \tau)$ does not contain an isometric copy of $L^{1}[0,1]$.

(7) $\mathcal{A}$ is $\tau$-atomic.

Proof. (7) $\Longrightarrow(1)$ If $L^{1}(\mathcal{A}, \tau)$ does not have the Radon Nikodym property, then $(s(\tau) \mathcal{A})_{*} \cong L^{1}(\mathcal{A}, \tau)$ also fails to have the Radon Nikodym property. Hence by [36, Theorem 3.5], there is a nonzero projection $P$ in $s(\tau) \mathcal{A} \subset \mathcal{A}$ which dominates no minimal projection. So $\mathcal{A}$ is not $\tau$-atomic.

$(1) \Longrightarrow(2)$ is well known (see [8]).

$(2) \Longrightarrow(3)$ Replacing $\mathcal{A}$ by $s(\tau) \mathcal{A}$, we may assume that $\tau$ is faithful on $\mathcal{A}$. If $L^{1}(\lambda, \tau) \cong \mathcal{A}_{*}$ has the Radon Nikodym property, let $S$ be the set of normal states in $\mathcal{A}_{*}$, and ext $(S)$ be the set of its extreme points. Then as in the proof of Theorem 4 in [9], if for each $f \in \operatorname{ext}(S),\left\{\pi_{f}, H_{f}\right\}$ is the representation of $\mathcal{A}$ induced by the GNS-construction, $\mathcal{A}$ is isomorphic to the direct sum $\sum \oplus B\left(H_{f}\right)$ taken over all $f \in \operatorname{ext}(S)$, where $B\left(H_{f}\right)$ denotes the set of all bounded linear operators from the Hilbert space $H_{f}$ into itself. In particular, by the uniqueness of the predual of a von Neumann algebra [35, p.135], $\mathcal{A}_{*}$ is isomorphic to the $\ell^{1}$-sum $\sum \oplus J\left(H_{f}\right)$, $f \in \operatorname{ext}(S)$, where $J\left(H_{f}\right)$ denotes the space of trace-class operators on $H_{f}$, which can be embedded as a subspace of $J(H)$, where $H$ is the Hilbert space direct sum of $H_{f}, f \in \operatorname{ext}(S)$. By a result of Lennard [27, $J(H)$ has UKK. In particular, every subspace of $J(H)$ has UKK. Hence (3) holds.

Implications $(3) \Longrightarrow(4) \Longrightarrow(5)$ are well known (see [13]).

That (5) implies (6) follows from 1 .

(6) $\Longrightarrow(7)$ Suppose $\mathcal{A}$ is not $\tau$-atomic. Then there is a projection $Q \in \mathcal{A}$ with $\tau(Q)>0$ which does not dominate any minimal projection. The projection $P=s(\tau) Q$ has the same properties and $\tau$ is faithful on $s(\tau) \mathcal{A}$, so by Lemma 3.1 $L^{1}(\mathcal{A}, \tau)=L^{1}(s(\tau) \mathcal{A}, \tau)$ contains an isometric copy of $L^{1}[0,1]$, i.e., (6) fails to hold.

Remark 3.5. Since $\tau$-atomicity of $\mathcal{A}$ amounts to atomicity of $s(\tau) \mathcal{A}$, the equivalence of (1) and (7) is already in [36], Theorem 3.5, and so is the further equivalent condition.

(8) $L^{1}(\mathcal{A}, \tau)$ is not a flat Banach space,

and, if $L^{1}(\mathcal{A}, \tau)$ is separable, the still equivalent condition

(9) $L^{1}(\mathcal{A}, \tau)$ is a dual space.

(Here conditions (1) - (8) imply (9) without separability assumption, so separability is only needed for the reverse implication.) The equivalence of (1) and (2) for preduals of arbitrary of Neumann algebras is due to 9 . 
Corollary 3.6. Let $\mathcal{A}$ be a von Neumann algebra. Then $\mathcal{A}$ is atomic if and only if $\mathcal{A}$ has a faithful semifinite normal trace and $\mathcal{A}_{*}$ has the weak fixed point property.

Proof. If $\mathcal{A}$ is atomic and $P$ is a nonzero central projection such that $P \mathcal{A}$ is the type III part of $\mathcal{A}$, then there exists a minimal projection $Q \leq P$. In particular $P \mathcal{A}$ contains a nonzero finite projection, which is impossible. So $\mathcal{A}$ is semifinite. Hence there is a faithful normal semifinite trace on $\mathcal{A}$ [35, p. 317]. By Proposition 3.4 above $\mathcal{A}_{*}$ has the weak fixed point property since for faithful $\tau$, atomic and $\tau$-atomic are the same.

The converse follows directly from Lemma 3.1.

Remark 3.7. For $1<p<\infty, \tau$ a faithful semifinite normal trace on a von Neumann algebra $\mathcal{A}$ or a faithful normal state on $\mathcal{A}$, then the Banach spaces $L^{p}(\mathcal{A}, \tau)$ is uniformly convex (see [31, 25] and 20, for definitions) using Theorem 4.2 in the Appendix of [20]. Consequently, by [7, $L^{p}(\mathcal{A}, \tau)$ has the weak fixed point property.

In the commutative case, Proposition 3.4 can be elaborated a bit, and the assumptions of semifiniteness and localization can be dropped:

Proposition 3.8. Let $(X, \mathcal{F}, \mu)$ be a measure space. The following are equivalent:

(1) $L^{1}(X, \mathcal{F}, \mu) \cong \ell^{1}(I)$ isometrically for some index set $I$.

(2) $L^{1}(X, \mathcal{F}, \mu)$ has the Radon Nikodym property.

(3) $L^{1}(X, \mathcal{F}, \mu)$ has the Krein Milman property.

(4) $L^{1}(X, \mathcal{F}, \mu)$ has the property UKK.

(5) $L^{1}(X, \mathcal{F}, \mu)$ has weak normal structure.

(6) $L^{1}(X, \mathcal{F}, \mu)$ has the weak fpp.

(7) $L^{1}(X, \mathcal{F}, \mu)$ does not contain an isometric copy of $L^{1}[0,1]$.

(8) $(X, \mathcal{F}, \mu)$ is atomic, i.e., every $A \in \mathcal{F}$ with $\mu(A)>0$ contains a $\mu$-atom.

Proof. (1) $\Longrightarrow(2)$ follows from [36, Theorem 3.5, since counting measure on $I$ is localizable.

$(2) \Longrightarrow(3)$ is well known (see [10]).

$(3) \Longrightarrow(4)$ : The set $A$ of all integrable $f \geq 0$ with $\int f d \mu=1$ is bounded closed and convex. The set of extreme points of $A$ is $E=\left\{\frac{1}{\mu(B)} 1_{B} \mid B \in \mathcal{F}\right.$, $B$ is a $\mu$-atom\}. By (3), $A$ is the closed convex hull of $E$. So for $f \in A$ there is a sequence of linear combinations $f_{n}=\sum_{i=1}^{k_{n}} \alpha_{i, n} 1_{B_{i, n}}$ with $\frac{1}{\mu\left(B_{i, n}\right)} 1_{B_{i, n}} \in E$ and $\left\|f_{n}-f\right\|_{1} \rightarrow 0$. Since only a countable number of $B_{i, n}$ is involved, we may represent all $f_{n}$ over one common sequence of (pairwise disjoint) $\mu$-atoms : $f_{n}=\sum_{i=1}^{\infty} \alpha_{i, n} 1_{B_{i}}$ almost everywhere, where for each $n$ only finitely many $\alpha_{i, n}$ are nonzero. We have $\alpha_{i, n}=\frac{1}{\mu\left(B_{i}\right)} \int f_{n} 1_{B_{i}} d \mu \rightarrow \frac{1}{\mu\left(B_{i}\right)} \int f 1_{B_{i}} d \mu \stackrel{\text { def }}{=} \alpha_{i}$, so $f=\sum_{1}^{\infty} \alpha_{i} 1_{B_{i}}$ a.e. since the $B_{i}$ are $\mu$-atoms. More generally, we have such a representation with $\sum_{1}^{\infty}\left|\alpha_{i}\right| \mu\left(B_{i}\right) \leq 1$ for every $f$ in the unit ball $B$ of $L^{1}(X, \mathcal{F}, \mu)$. Now, to prove (4), let $\varepsilon>0$ and $g, g_{n} \in B$ with $g_{n} \rightarrow g$ weakly and $\inf _{n \neq m}\left\|g_{n}-g_{m}\right\|_{1}>\varepsilon$. We want to show $\|g\|_{1} \leq 1-\frac{\varepsilon}{4}$. Again, we may assume a common representation over disjoint $\mu$-atoms, $g_{n}=\sum_{i=1}^{\infty} \beta_{i, n} 1_{B_{i}}$ a.e. and $g=\sum_{i=1}^{\infty} \beta_{i} 1_{B_{i}}$, with $\sum_{i=1}^{\infty}\left|\beta_{i, n}\right| \mu\left(B_{i}\right) \leq 1$ for all $n$, and $\beta_{i, n} \rightarrow \beta_{i}$ as $n \rightarrow \infty$. 
Assume $\|g\|_{1}>1-\frac{\varepsilon}{4}$ and choose $i_{0} \in \mathbb{N}$ such that $\sum_{1}^{i_{0}}\left|\beta_{i}\right| \mu\left(B_{i}\right)>1-\frac{\varepsilon}{4}$ and $n_{0} \in \mathbb{N}$ such that $\sum_{i=1}^{i_{0}}\left|\beta_{i, n}-\beta_{i}\right| \mu\left(B_{i}\right)<\frac{\varepsilon}{8}$ and $\sum_{i=1}^{i_{0}}\left|\beta_{i, n}\right| \mu\left(B_{i}\right)>1-\frac{\varepsilon}{4}$ for $n \geq n_{0}$. Then

$$
\begin{aligned}
\left\|g_{n_{0}}-g_{n_{0}+1}\right\|_{1} a m p & ;=\sum_{i=1}^{i_{0}}\left|\beta_{i, n_{0}}-\beta_{i, n_{0}+1}\right| \mu\left(B_{i}\right)+\sum_{i>i_{0}}\left|\beta_{i, n_{0}}-\beta_{i, n_{0}+1}\right| \mu\left(B_{i}\right) \\
a m p & ; \leq 2 \cdot \frac{\varepsilon}{8}+\left(\frac{\varepsilon}{4}+\frac{\varepsilon}{4}\right)<\varepsilon
\end{aligned}
$$

a contradiction. So $L^{1}(X, \mathcal{F}, \mu)$ satisfies the property UKK with $\delta=\frac{\varepsilon}{4}$.

$(4) \Longrightarrow(5) \Longrightarrow(6)$ is known (see [13]).

$(6) \Longrightarrow(7)$ follows from [1.

$(7) \Longrightarrow(8)$ was proved in Proposition 3.3.

$(8) \Longrightarrow(1)$ :

Suppose every $A \in \mathcal{F}$ with $0<\mu(A)<\infty$ contains a $\mu$-atom. Using Zorn's Lemma we can show that every such $A$ is the almost disjoint union of $\mu$-atoms (the intersection of two atoms has measure zero). It follows that every $f \in \mathcal{L}^{1}(\mu)$ is equivalent to a countable sum $\sum \lambda_{i} 1_{A_{i}}$ with suitable $\lambda_{i}$ and $\mu$-atoms $A_{i}$ and $\sum\left|\lambda_{i}\right| \mu\left(A_{i}\right)=\|f\|_{1}$. The $A_{i}$ in such a representation are unique up to sets of measure zero. Now let $I$ be a complete set of representatives of the set of all $\mu$-atoms in $X$ for the equivalence relation $\sim$, where $B \sim C$, if $\mu(B \Delta C)=0$. The map $\left(\alpha_{B}\right)_{B \in I} \mapsto \sum_{B \in I} \alpha_{B} \frac{1}{\mu(B)} 1_{B}$ is an isometric isomorphism from $\ell^{1}(I)$ onto $L^{1}(\mu)$.

Remark 3.9. Since from 34, p. 275 and p. 301, one can see that every $L^{1}(X, \mathcal{F}, \mu)$ is the predual of a (semifinite) von Neumann algebra (not necessarily $L^{\infty}(X, \mathcal{F}, \mu)$; see [26, p. 108), we may again add in Proposition 3.8 the equivalent condition

(9) $L^{1}(X, \mathcal{F}, \mu)$ is not a flat Banach space.

The conditions (1) - (9) all imply the following condition and, if $L^{1}(X, \mathcal{F}, \mu)$ is separable, are equivalent to it:

(10) $L^{1}(X, \mathcal{F}, \mu)$ is a dual space.

Let us also remark that, for the reason mentioned a few lines above, most of Proposition 3.8 could be concluded from the von Neumann algebra result Proposition 3.4, but the reader may find it convenient to read measure theoretic arguments for measure space results.

Finally, we note another condition which is equivalent to (1) - (9):

(11) Every weakly convergent sequence in $L^{1}(X, \mathcal{F}, \mu)$ is norm convergent.

The implication (1) $\Rightarrow(11)$ is Schur's Theorem (see [37, p. 122). If (11) holds, no sequence can satisfy the hypothesis of UKK, so $(11) \Rightarrow(4)$ holds.

Remark 3.10. Weights do not matter for the weak fpp except that they transfer the problem to a measure subspace: If $\omega$ is a weight on the measure space $(X, \mathcal{F}, \mu)$, consider $A=\{0<\omega<\infty\}, \mathcal{F}_{A}=\{F \cap A \mid F \in \mathcal{F}\}$, and $\mu_{A}=\left.\mu\right|_{\mathcal{F}_{A}}$. Then $L^{1}(X, \mathcal{F}, \omega \mu) \cong L^{1}\left(A, \mathcal{F}_{A}, \omega \mu_{A}\right)$, and the last space is isometrically isomorphic to $L^{1}\left(A, \mathcal{F}_{A}, \mu_{A}\right)$ by the map $f \mapsto \omega f$. So $L^{1}(X, \mathcal{F}, \omega \mu)$ has the weak fpp if and only if the measure space $\left(A, \mathcal{F}_{A}, \mu_{A}\right)$ is atomic. 
In particular, if $X$ is some (Lebesgue) measurable subset of $\mathbb{R}^{n}, \mathcal{F}$ is the collection of measurable subsets of $X$, and $\mu$ is Lebesgue measure, then $L^{1}(X, \mathcal{F}, \omega \mu)$ has the weak fpp if and only if it is zero, which is equivalent to $\mu(A)=0$. On the other hand, for any weight $\omega$ on a nonvoid set $\Gamma$, the space $\ell^{1}(\Gamma, \omega)$ has the weak fpp.

A dual Banach space $E$ is said to have the weak ${ }^{*}$-fixed point property (weak ${ }^{*}$ fpp) if any weak ${ }^{*}$-compact convex subset $K \subseteq E$ has the fixed point property. It was shown by $\operatorname{Lim}\left[28\right.$ ] that $\ell^{1}$ has the weak ${ }^{*}$ fpp. The following is a strengthening of Lim's result and Remark 3.10 .

Proposition 3.11. For any nonempty set $\Gamma$ and weight $\omega$ on $\Gamma, \ell^{1}(\Gamma, \omega)$ has the weak ${ }^{*}$ fpp.

Proof. We know that $\ell^{1}(\Gamma)$ satisfies Lim's condition [28] (see also 22]), i.e., whenever $f_{\alpha}$ is a net in $\ell^{1}(\Gamma)=c_{0}(\Gamma)^{*}$, with $f_{\alpha} \rightarrow 0$ in the weak ${ }^{*}$-topology, and $\lim _{\alpha}\left\|f_{\alpha}\right\|=s$, then

$$
\lim _{\alpha}\left\|f_{\alpha}+g\right\|=s+\|g\|
$$

for all $g \in \ell_{1}(\Gamma)$. It follows that $\ell^{1}(\Gamma, \omega)$ also satisfies Lim's condition. So $\ell^{1}(\Gamma, \omega)$ has the RNP and weak ${ }^{*}$-normal structure (Lemma 4 in [22]). Consequently it has the weak ${ }^{*}$ fpp [28].

\section{Fourier Algebra, Fourier-Stieltjes ALGEBRA AND WEAK FPP}

Let $G$ be a locally compact group. We define $C^{*}(G)$, the group $C^{*}$-algebra of $G$, to be the completion of $L^{1}(G)$ with respect to the norm

$$
\|f\|_{*}=\sup \left\|\pi_{f}\right\|,
$$

where the supremum is taken over all nondegenerate $*$-representations $\pi$ of $L^{1}(G)$ as an $*$-algebra of bounded operators on a Hilbert space. Let $C(G)$ be the Banach space of bounded continuous complex-valued functions on $G$ with the supremum norm. Denote the set of continuous positive definite functions on $G$ by $P(G)$, and the set of continuous functions on $G$ with compact support by $C_{00}(G)$. Define the Fourier-Stieltjes algebra of $G$, denoted by $B(G)$, to be the linear span of $P(G)$. The Fourier algebra of $G$, denoted by $A(G)$, is defined to be the closed linear span of $P(G) \cap C_{00}(G)$. Clearly, $A(G)=B(G)$ if $G$ is compact. Let $\lambda$ be the left regular representation of $G$, i.e., for each $f \in L^{1}(G), \lambda(f)$ is the bounded operator in $\mathcal{B}\left(L^{2}(G)\right)$ defined by $\lambda(f)(h)=f * h$ (the convolution of $f$ and $h$ ). Then denote by $V N(G)$ the closure of $\left\{\lambda(f): f \in L^{1}(G)\right\}$ in the weak operator topology in $\mathcal{B}\left(L^{2}(G)\right.$ ). It is known that $C^{*}(G)^{*}=B(G)$ and $A(G)^{*}=V N(G)$ (see [14 for details).

$G$ is called an [IN]-group if there is a compact neighbourhood of the identity $e$ in $G$ which is invariant under inner automorphisms; $G$ is a [SIN]-group if there is a base for the neighbourhood system of $e$ consisting of compact sets invariant under inner automorphisms (or equivalently, the left and right uniformities on $G$ are the same; see $[32])$. Obviously [compact $] \subseteq[\mathrm{SIN}] \subseteq[\mathrm{IN}]$ and the inclusions are proper. Furthermore, all [IN]-groups are unimodular (see [32]). 
Theorem 4.1. Let $G$ be a locally compact group with semifinite von Neumann algebra $V N(G)$ (e.g. $G$ unimodular). The following are equivalent:

(1) $A(G)$ has the Radon Nikodym Property.

(2) $A(G)$ has the Krein Milman Property.

(3) $A(G)$ has UKK.

(4) $A(G)$ has weak normal structure.

(5) $A(G)$ has the weak fixed point property.

(6) $A(G)$ does not contain an isometric copy of $L^{1}[0,1]$.

(7) $V N(G)$ is atomic.

(8) $A(G)$ is not a flat Banach space.

The preceding conditions imply and, if $G$ is separable, are equivalent to

(9) $A(G)$ is a dual space.

Proof. Since $V N(G)$ is a semifinite von Neumann algebra, it has a faithful semifinite normal trace. Apply now Proposition 3.4 and Remark 3.5 .

The following corollary improves Theorem 3.5 and Proposition 3.6 in [23]:

Corollary 4.2. Let $G$ be an [IN]-group. Then $A(G)$ has the weak fixed point property (respectively RNP, KMP, UKK, weak normal structure and so on) if and only if $G$ is compact.

Proof. This follows from Theorem 4.1 and [24, Theorem 4.3].

Proposition 4.3. Let $G$ be a locally compact group. If $A(G)$ has the RNP, then $A(G)$ has the weak fpp for commuting semigroups of nonexpansive mappings.

Proof. This follows from the fact that $A(G)$ has the weak fpp [23] and 8$]$.

Remark 4.4. Examples of locally compact groups $G$ such that $A(G)$ has the RNP include the " $a x+b$ " group which is solvable (hence amenable) but nonunimodular. (See [36] and [2] for details.) This shows that the assumption [IN] in Corollary 4.2 is not superfluous.

In the conditions (4) - (8) of the following theorem one may replace " $G$ is type I" by " $W^{*}(G)$ is semifinite", as will be seen from the proof.

Theorem 4.5. Let $G$ be a locally compact group. The following are equivalent:

(1) $B(G)$ has the Radon Nikodym property.

(2) $B(G)$ has the Krein Milman property.

(3) The von Neumann algebra $W^{*}(G)=B(G)^{*}$ is atomic.

(4) $G$ is type $I$, and $B(G)$ has UKK.

(5) $G$ is type $I$, and $B(G)$ has weak normal structure.

(6) $G$ is type $I$, and $B(G)$ has the weak fixed point property.

(7) $G$ is type $I$, and $B(G)$ does not contain an isometric copy of $L^{1}[0,1]$.

(8) $G$ is type $I$, and $B(G)$ is not a flat Banach space.

(9) $G$ is type $I$, and $C^{*}(G)$ does not contain an isometric copy of $\ell^{1}$.

Proof. (1) $\Longleftrightarrow(2)$ is in [7, and (1) $\Longleftrightarrow(3)$ follows from [34, Theorem 3.5]. The equivalence of conditions (1) - (8) is a consequence of Proposition 3.4 and the following: If $B(G)$ has the RNP, by [34, Theorem 4.4] $G$ must be type I. If $G$ is type I, $W^{*}(G)$ has a faithful semifinite normal trace. 
(1) $\Longrightarrow$ (9) follows from [36, Theorem 4.4] and Stegall's Theorem [10, p. 195].

(9) $\Longrightarrow$ (6) follows from [23, Corollary 3.2].

Corollary 4.6. Let $G$ be an almost connected Type I group. Then $B(G)$ has the weak fixed point property if and only if $G$ is compact.

Proof. This follows from [36, Theorem 4.11] and the above.

\section{Fourier ALGEBRA AND FPP}

In this section, we shall study the fpp for the Fourier algebra $A(G)$ of a locally compact group $G$ and its closed ideals. We begin with the following useful observation.

Proposition 5.1. If a nonzero closed ideal $I$ of $A(G)$ has the fpp, then $G$ has arbitrarily small compact open subgroups, i.e., $G$ is totally disconnected.

Proof. For $G$ discrete, there is nothing to prove; so we may assume that $G$ is nondiscrete. Let $I$ be a nonzero closed ideal of $A(G)$ having the fpp, and let $U$ be a neighbourhood of $e$. Since $I \neq 0$, there is a point $x$ outside the zero set $Z(I)=\{y \in$ $G \mid f(y)=0$ for all $f \in I\}$. Using a translation (which is a linear isometry) we may suppose $x=e$. There is a positive definite function $t$ with $t(e)=1$ and compact support contained in $U \cap(G \backslash Z(I))$ (see [14]). Let $K=\{g \in I \mid g$ positive definite, $g(e)=1\}$. Then $K$ is a nonempty (e.g. $t \in K$ ) bounded norm closed convex of $I$. Define $T: K \rightarrow K$ by $T g=t \cdot g$. Since $\|T f-T g\|_{A(G)} \leq\|f-g\|_{A(G)}$, by assumption, there is a fixed point $h \in K: T h=h$. Since $h$ is nonzero in a neighbourhood $V$ of $e$, we have $t=1$ on $V$, so the peak set $H=\{y \in G \mid t(y)=t(e)=1\}$ is an open (and hence closed) subgroup of $G$ contained in supp $t$.

So $H$ is a compact open subgroup contained in $U$. Since $U$ was arbitrarily chosen, the assertion follows.

Lemma 5.2. Let $V \subset G$ be nonclosed and suppose $x \in \bar{V} \backslash V$. For any neighbourhood $Z$ of e, there is some $z \in Z$ with $x \in z V$.

Proof. Since $x \in \bar{V} \backslash V$, the set $Z^{-1} x$ intersects $V$, so there is some $v \in V \cap Z^{-1} x$. In particular $z v=x$ for some $z \in Z$, which proves the assertion.

Lemma 5.3. Let $G$ be first countable totally disconnected nondiscrete. For every open e-neighbourhood $U$ there is an open subset $V \subset U$ with $|V|>\frac{2}{3}|U|$ and such that $e \in(\bar{V} \backslash V) \cap(\overline{U \backslash \bar{V}})$.

Proof. Choose a strictly decreasing sequence of compact open $e$-neighbourhoods $V_{i} \subset U$ with $\left|V_{1}\right|<\frac{1}{3}|U|$ and $\bigcap_{i} V_{i}=\{e\}$. Choose $U_{i} \subset V_{i} \backslash V_{i+1}$ compact open nonvoid with $\left|U_{i}\right|<\frac{1}{2}\left|V_{i} \backslash V_{i+1}\right|$. Let $V=U \backslash\left(\overline{\bigcup_{i} U_{i}}\right)$. Then $V$ is nonvoid open with $|V|>\frac{2}{3}|U|$ and $e \notin V$ since $e \in \overline{U U_{i}}$ : if $y_{i} \in U_{i}$, then $y_{i} \rightarrow e$ since $U_{i} \subset V_{i}$.

Since $\left(V_{i} \backslash V_{i+1}\right) \backslash\left(\overline{\bigcup U_{j}}\right)=\left(V_{i} \backslash V_{i+1}\right) \backslash U_{i}$ is nonvoid for all $i$, we have $e \in \bar{V}$ : if $x_{i} \in\left(V_{i} \backslash V_{i+1}\right) \backslash\left(\overline{\bigcup_{j} U_{j}}\right)$, then $x_{i} \rightarrow e$. But also $e \in \overline{U \backslash \bar{V}}$ since (as seen above) $e \in \overline{U U_{i}}$ and $\bigcup U_{i} \subset U \backslash \bar{V}$.

If $f$ is a function on $G$, we write $f^{\vee}(x)=f\left(x^{-1}\right), x \in G$. 
Lemma 5.4. Let $G$ be first countable totally disconnected nondiscrete. Let $U$ be an open compact subgroup of $G$ and let $V \subset U$ be as in Lemma 5.3. Then the function $t=1_{V} * 1_{V}^{\vee}$ has its support equal to $U$ and is not constant in any neighbourhood of $e$.

Proof. Clearly $t=0$ outside $U$. For $v \in U$ we have

$$
t(v)=\int 1_{V}(v y) 1_{V}(y) d y=\left.\right|_{v^{-1}} V \cap V\left|>\frac{1}{3}\right| U \mid>0
$$

since both of the intersecting sets have Haar measure $>\frac{2}{3}|U|$. Now let $Z$ be some symmetric neighbourhood of $e$. For $v=z^{-1}$, where $z \in Z$ is as in Lemma 5.2 (with $x=e$ ), we have $e \in v^{-1} V$. By Lemma $5.3 v^{-1} V$ must also contain a point from $U \backslash \bar{V}$, i.e., an exterior point $s$ of $V$. This means that $\left|{ }_{v^{-1}} V \cap V\right|<|V|$, since $v^{-1} V$ contains a whole neighbourhood of $s$ which lies in the complement of $V$. Thus we have $t(v)<t(e)$ for this $v \in Z$. So $t$ is not constant on $Z$.

Theorem 5.5. Let $G$ be totally disconnected and nondiscrete. Then every nonzero closed ideal I of $A(G)$ fails the fpp.

Proof. Let $I$ be a nonzero closed ideal in $A(G)$. Since $I \neq\{0\}$, the open set $G \backslash Z(I)$ is nonvoid. By translating, if necessary, we may assume $e \in G \backslash Z(I)$. Let $B \subset G$ be a compact open subgroup. Since $1_{B} \in B(G)$, the set $J=I \cdot 1_{B}=\left\{f \cdot 1_{B} \mid f \in I\right\}$ is a closed nonzero ideal contained in $I$. It suffices to show that $J$ fails the norm fpp. The restriction map $\left.g \mapsto g\right|_{B}$ is an isometric isomorphism from $J$ onto a closed nonzero ideal $J^{\prime} \subset A(B)$ with zero set $Z\left(J^{\prime}\right)=Z(I) \cap B$. This reduces the problem to a compact group. Let us from now on assume that $G$ is compact. Let $\left(U_{i}\right)$ be a sequence of strictly decreasing compact open subgroups of $G$ with $\left|U_{i}\right| \rightarrow 0$. By [16. Theorem 8.7, p. 71] there is a compact normal subgroup $H \subset \bigcap_{i} U_{i}$ such that $G / H$ is separable.

Since $|H| \leq\left|U_{i}\right|$ for all $i$, we have $|H|=0$, so $H$ is not open and hence $\dot{G}=G / H$ is nondiscrete. In the proof of the quoted Theorem 8.7 in [16], we may choose the $V_{i} \subset U_{i}$ to be compact open subgroups, with the effect that $\dot{G}$ is then totally disconnected, since the identity component $C$ of $\dot{e}$ satisfies $C \subset \bigcap_{i} \dot{V}_{i}=H=\{\dot{e}\}$ (see [16, Theorem 7.8, p. 62]). So $\dot{G}$ satisfies the assumptions made for $G$ in Lemma 5.4. Since $\dot{G}$ has finite topological dimension, there is a cross-section $\sigma: \dot{G} \rightarrow G$ (i.e., $\sigma(\dot{x}) \in \dot{x}$ for every $\dot{x} \in \dot{G}$ ) which is continuous on an open neighbourhood $x$ of $\dot{e}$ (see [30]). Since $e \in G \backslash Z(I)$, there are open identity neighbourhoods $U \subset X \subset \dot{G}$ and $Y \subset H$ such that " $U \times Y$ " $\subset G \backslash Z(I)$ (i.e., $\{\sigma(\dot{x}) y \mid \dot{x} \in U, y \in Y\} \subset G \backslash Z(I)$ ). Let $f \in A(G)$ be positive definite with $f(e)=1$ and compact support contained in $U \times Y \subset G \backslash Z(I)$. Define $K=\{f \cdot(h \circ p) \mid h \in P(\dot{G}) \cap K(G), h(\dot{e})=1, \operatorname{supp} h \subset U\}$ where $p: x \mapsto \dot{x}$ is the canonical projection from $G$ onto $\dot{G}$. Then $K \subset I$ is bounded convex, so $\bar{K}$ is bounded norm closed convex in $I$. (We remind the reader that functions in $A(G)$ with compact support disjoint from $Z(I)$ belong to $I$ independent of whether or not $Z(I)$ has synthesis. The disjointness is guaranteed by $f$ above.) Let $s=\frac{1}{|V|} t$, where $t$ is a function like in Lemma 5.4 on $\dot{G}$ with support in $U$, and define $T: \bar{K} \rightarrow \bar{K}$ by $T g=(s \circ p) \cdot g$. Since $s \circ p(e)=1, T$ is well-defined and does not increase norms. There is no fixed point for $T$ in $\bar{K}$, because otherwise $s \circ p$ and hence $s$ would have to be equal to one in a neighbourhood of the identity, which is not possible by Lemma 5.4. So $I$ fails the norm fpp. 
Corollary 5.6. For $G$ locally compact, if a nonzero closed ideal of $A(G)$ has the fpp, then $G$ is discrete.

Proof. Proposition 5.1 and Theorem 5.5.

Theorem 5.7. $A(G)$ has the fpp if and only if $G$ is finite.

Proof. Suppose $A(G)$ has the fpp. By Corollary 5.6, $G$ is discrete. Since the fpp implies the weak fpp, $G$ is compact by Corollary 4.2 . So $G$ is finite. The converse is clear, since $A(G)$ is finite dimensional, if $G$ is finite.

Corollary 5.8. $B(G)$ has the fpp if and only if $G$ is finite.

Proof. Since $A(G)$ is a closed subspace of $B(G)$, the assertion follows from Theorem 5.7.

For $g \in G$ we denote by $G(g)$ the subgroup generated by $g$.

Proposition 5.9. Let $G$ be discrete and I a nonzero closed ideal of $A(G)$. If there is an element $g \in G$ of infinite order such that the intersection of the zero set $Z(I)$ with some coset of $G(g)$ is finite (possibly empty), then I does not have the fpp.

Proof. Suppose $Z(I) \cap a G(g)=F$ is finite for some $a \in G$. Then the ideal $I\left(a^{-1} F\right)=$ $\left\{h \in A(G(g))|h|_{a^{-1} F}=0\right\}$ in $A(G(g)) \cong A(\mathbb{Z})$ is not reflexive, as it has finite codimension and $A(\mathbb{Z}) \cong L^{1}[0,1]$ is not reflexive. By [12, $I\left(a^{-1} F\right)$ fails the fpp. Since $I\left(a^{-1} F\right)$ is canonically and isometrically included in $A(G)$ (extend by 0 outside $G(g)$ ), we may consider it as an ideal in $A(G)$. It is isometrically isomorphic to $I(F)$ by translation with $a$. Since $I(F) \subset I$, the assertion follows. (For this last conclusion we implicitly used the fact that subsets of $\mathbb{Z}$ have synthesis: every $f \in I\left(a^{-1} F\right)$ can be approximated in norm by $h \in A(\mathbb{Z})$ with finite support disjoint from $a^{-1} F$. This carries over to $I\left(a^{-1} F\right)$ and $I(F)$ as ideals of $A(G)$. Any function with finite support disjoint from $Z(I)$ belongs to $I$. Since every $f \in I(F)$ is the limit of such functions and $I$ is closed, we have $I(F) \subset I$ ).

Corollary 5.10. If $G$ is a discrete group with an element of infinite order, then maximal ideals in $A(G)$ and finite intersections of them do not have the fpp.

Proposition 5.11. Let $G$ be a discrete group and $I$ be a nonzero closed ideal of $A(G)$ as in Proposition 5.9 or Corollary 5.10 failing the fpp. Then there exists a (closed) infinite dimensional ideal $J \subseteq I$ such that $J$ has the fpp.

Proof. In the proof of Proposition 5.9 we used an ideal of $A(G(g)) \cong A(\mathbb{Z})$ with finite zero set. By [33] or [6], the complement of this set in $\mathbb{Z}$ contains a $\Lambda(p)$ set for all finite $p$, so that for functions supported on this set, the norms in $A(G)$ and in $\mathcal{L}^{2}$ are equivalent. The set of these functions is a reflexive ideal in $A(\mathbb{Z}) \subset A(G)$ contained in $I$, and by [29] has the fpp.

Proposition 5.12. Let $G$ be a countable discrete abelian group. A closed ideal (or even a closed subspace) I of $A(G)$ has the fpp if and only if $I$ is reflexive.

Proof. We have $A(G) \cong \mathcal{L}^{1}(\widehat{G})$ where $\widehat{G}$ is the dual group. $\widehat{G}$ is compact. Since $G$ is countable, $A(G)$ and hence $\mathcal{L}^{1}(\widehat{G})$ are separable. So $(\widehat{G}, \lambda)$ is separable, where $\lambda$ is the normalized Haar measure on the Borel sets of $\widehat{G}$. If $\widehat{G}$ is discrete, then it is finite, and so is $G$; hence the assertion holds. If $\widehat{G}$ is nondiscrete, $(\widehat{G}, \lambda)$ does not have any atoms by [16, Theorem 11.44]. By [15, Theorem C, p. 173] and an 
additional argument we have $\mathcal{L}^{1}(\widehat{G}) \cong \mathcal{L}^{1}[0,1]$ isometrically, so the assertion follows by [19].

Remark 5.13. In the case of Proposition 5.12, if $I$ fails the fpp, there is an infinite dimensional (closed) ideal $J \subset I$ having the fpp (see the proof of Proposition 5.11).

\section{REFERENCES}

[1] D. Alspach, A fixed point free nonexpansive map, Proc. Amer. Math. Soc. 82 (1981), 423-424. MR612733 (82j:47070)

[2] L. Baggett and K. Taylor, Groups with completely reducible regular representation, Proceedings American Mathematical Society 72 (1978), 593-600. MR509261 (80b:22009)

[3] T.D. Benavides, M.A. Japon Pineda and S. Prus, Weak compactness and fixed point property for affine maps, Journal of Functional Analysis 209 (2004), 1-15. MR2039215 (2005b:46046)

[4] T.D. Benavides and M.A. Japon Pineda, Fixed points of nonexpansive mappings in spaces of continuous functions, PAMS 133 (2005), 3037-3046. MR2159783 (2006d:47097)

[5] R. Bourgin, Geometric aspects of convex sets with the Radon-Nikodym property, Lecture Notes in Math. 993, Springer-Verlag, Berlin, 1983. MR704815 (85d:46023)

[6] M. Bozejko, The existence of $\lambda(p)$ sets in discrete noncommutative groups, Boll. Un. Mat. Ital. (4) 8 (1973), 579-582. MR0344805 (49:9544)

[7] F.E. Browder, Nonexpansive nonlinear operators in Banach spaces, Proc. Nat. Acad. Sci. U.S.A. 54 (1965), 1041-1044. MR0187120 (32:4574)

[8] R.E. Bruck, A common fixed point theorem for a commutative family of nonexpansive mappings, Pacific Journal of Mathematics 53 (1974), 59-71. MR0361945 (50:14387)

[9] C.H. Chu, A note on scattered $C *$-algebras and the Radon-Nikodym property, J. London Math. Soc. 24 (1981), 533-536. MR635884 (82k:46086)

[10] J. Diestel and J.J. Uhl, Jr., Vector measures, Mathematical Survey 15, Amer. Math. Society, 1977. MR0453964 (56:12216)

[11] J. Dixmier, $C^{*}$-algebras, North-Holland Publishing Co., New York, 1977. MR0458185 $(56: 16388)$

[12] P.N. Dowling, C.J. Lennard and B. Turett, The fixed point property for subsets of some classical Banach spaces, Nonlinear Analysis, Theory, Methods and Applications 49 (2002), 141-145. MR 1887917 (2002k:47115)

[13] D. van Dulst and B. Sims, Fixed points of nonexpansive mappings and Chebyshev centers in Banach spaces with norms of type (KK), p. 35-43 in: Lecture Notes in Math. (Banach space theory and its applications, Bucharest, 1981), vol. 991, Springer-Verlag, New York, 1983. MR714171 (84i:46027)

[14] P. Eymard, L'algèbre de Fourier d'un groupe localement compact, Bull. Soc. Math. France 92 (1964), 181-236. MR0228628 (37:4208)

[15] P.R. Halmos, Measure Theory, Van Nostrand, 1950. MR0033869 (11:504d)

[16] E. Hewitt and K.A. Ross, Abstract Harmonic Analysis, vol. I, Springer-Verlag, 1963. MR.551496 (81k:43001)

[17] R. Huff, Banach spaces which are nearly uniformly convex, Rocky Mountain J. Math. 10 (1980), 743-749. MR595102 (82b:46016)

[18] W.A. Kirk, A fixed point theorem for mappings which do not increase distances, Amer. Math. Monthly 72 (1965), 1004-1006. MR0189009 (32:6436)

[19] W.A. Kirk and K. Goebel, Classical theory of nonexpansive mappings, in Handbook of Metric Fixed Point Theory, Kluwer, 49-91, 2001. MR.1904274 (2004c:47105)

[20] H. Kosaki, Applications of the complex interpolation method to a von Neumann algebra: Non-commutative $L^{p}$-spaces, Journal of Functional Analysis 56 (1984), 29-78. MR735704 (86a:46085)

[21] A.T. Lau and P.F. Mah, Normal structure in dual Banach spaces associated with a locally compact group, Trans. Amer. Math. Soc. 310 (1988), 341-353. MR937247 (89e:43004)

[22] A.T. Lau and P.F. Mah, Quasi-normal structures for certain spaces of operators on a Hilbert space, Pacific Journal of Mathematics 121 (1986), 109-118. MR815037 (87f:47065)

[23] A.T. Lau, P.F. Mah and A. Ülger, Fixed point property and normal structure for Banach spaces associated to locally compact groups, Proc. A.M.S. 125 (1997), 2021-2027. MR1372037 (97i:43001) 
[24] A.T. Lau and A. Ülger, Some geometric properties on the Fourier and Fourier-Stieltjes algebras of locally compact groups, Arens regularity and related problems, Trans. Amer. Math. Soc. 337 (1993), 321-359. MR1147402 (93g:22007)

[25] M. Leinert, On integration with respect to a trace, in Aspects of Positivity in Functional Analysis, North Holland Math. Stud. 122, 231-239, 1986. MR859732 (88b:46095)

[26] M. Leinert, Integration und Maß, Vieweg 1995. MR1396785 (97j:28001)

[27] C. Lennard, $C_{1}$ is uniformly Kadec-Klee, Proc. Amer. Math. Soc. 109 (1990), 71-77. MR943795 (90h:46029)

[28] T.C. Lim, Asymptotic centres and nonexpansive mappings in conjugate spaces, Pacific J. Math. 90 (1980), 135-143. MR.599326 (82h:47052)

[29] B. Maurey, Points fixes des contractions de certains faiblement compacts de $L^{1}$, Seminaire d'Analyse Functionnelle 80-81, Ecole Polytechnique, Palaiseau, 1981. MR659309 (83h:47041)

[30] P.S. Mostert, Sections in principal fibre spaces, Duke Math. J. 23 (1956), 57-71. MR0075575 $(17: 771 \mathrm{f})$

[31] E. Nelson, Notes on non-commutative integration, Journal of Functional Analysis 15 (1974), 103-116. MR.0355628 (50:8102)

[32] T.W. Palmer, Classes of nonabelian, noncompact, locally compact groups, Rocky Mountain J. of Math. 8 (1978), 683-741. MR513952 (81j:22003)

[33] M. Picardello, Lacunary sets in discrete noncommutative groups, Boll. Un. Math. Ital. (4) 8 (1973), 494-508. MR0344804 (49:9543)

[34] I.E. Segal, Equivalences of measure spaces, Amer. J. Math. 73 (1951), 275-313. MR0041191 $(12: 809 f)$

[35] M. Takesaki, Theory of Operator Algebras I, Springer-Verlag, New York, 1979. MR548728 (81e:46038)

[36] K. Taylor, Geometry of the Fourier algebras and locally compact groups with atomic unitary representations, Math. Ann. 262 (1983), 183-190. MR690194 (84h:43020)

[37] K. Yosida, Functional Analysis, Springer-Verlag, Berlin, Heidelberg, New York, 1978. MR0500055 (58:17765)

Department of Mathematical and Statistical Sciences, University of Alberta, Edmonton, Alberta, Canada T6G 2G1

E-mail address: tlau@math.ualberta.ca

Institut für Angewandte Mathematik, Universität Heidelberg, Im Neuenheimer Feld, Gebäude 294, 69120 Heidelberg, Germany

E-mail address: leinert@math.uni-heidelberg.de 